\title{
BMJ Open Tackling frailty at primary care: evaluation of the effectiveness of a multicomponent intervention through a randomised controlled trial: study protocol
}

\author{
Francisco Rivas-Ruiz (10 ,1,2 Mónica Machón, ${ }^{2,3,4}$ Maider Mateo-Abad, ${ }^{2,4}$ \\ Eugenio Contreras-Fernández, ${ }^{2,5}$ Carolina Güell, ${ }^{3,6}$ Luis Baro-Rodríguez, ${ }^{7}$ \\ Kalliopi Vrotsou, ${ }^{2,3,4}$ Raúl Quirós-López, ${ }^{2,8}$ Itziar Vergara (1) , 2,3,4 on behalf of the \\ InFrAP investigators
}

To cite: Rivas-Ruiz F, Machón M, Mateo-Abad M, et al. Tackling frailty at primary care: evaluation of the effectiveness of a multicomponent intervention through a randomised controlled trial: study protocol. BMJ Open 2020;10:e034591. doi:10.1136/ bmjopen-2019-034591

- Prepublication history for this paper is available online. To view these files, please visit the journal online (http://dx.doi. org/10.1136/bmjopen-2019034591).

Received 02 0ctober 2019 Revised 10 January 2020 Accepted 29 January 2020
D) Check for updates

(C) Author(s) (or their employer(s)) 2020. Re-use permitted under CC BY-NC. No commercial re-use. See rights and permissions. Published by BMJ.

For numbered affiliations see end of article.

Correspondence to Mónica Machón; monica.machonsobrado@ osakidetza.eus

\section{ABSTRACT}

Introduction This project focuses on how frailty is addressed in primary healthcare (PHC) and will evaluate the effectiveness of a multifactorial intervention (considering the appropriateness of the pharmaceutical prescription, the nutritional care provided and the exercise intervention) for persons with frailty, in terms of improving their functional capacity and reducing the incidence of adverse events related to frailty. The final evaluation will be made at 12 months' follow-up.

Methods and analysis Pragmatic multicentre cluster randomised controlled clinical trial, single blind with two arms: multifactorial intervention in PHC versus usual follow-up. The randomisation unit is the patient list and the analysis unit is the patient. In addition, a cost-effectiveness study and a qualitative study will be carried out, the latter based on semistructured interviews and focus groups. Two hundred persons (100 per study branch) all aged $\geq 70$ years, presenting frailty, but functionally independent and resident in the community, will be recruited. A baseline evaluation will be carried out prior to the intervention, with follow-up at 6 and 12 months. The main study variables considered will be functional capacity and incidence of adverse events; the secondary variables considered will be the patients' sociodemographic characteristics, nutritional status, level of physical activity and drug consumption, together with data on comorbidity, cognitive and affective status and health-related quality of life. Data will be analysed according to the intention-to-treat principle using a $5 \%$ significance level.

Ethics and dissemination The study will at all times be conducted in strict accordance with the provisions of the Declaration of Helsinki and with the national legislation regulating patients' autonomy. All patients recruited will be asked to provide written informed consent before taking part in the clinical trial. On completion of the study, the principal investigator expects to publish the results of this research in a peer-reviewed open access scientific journal.

Trial registration number ISRCTN17143761.

\section{Strengths and limitations of this study}

- Pragmatic multicentre clinical trial, single blind, cluster randomised, with two arms: multifactorial intervention in primary healthcare versus usual follow-up.

- The doctors and nurses collaborating in the fieldwork for this project will take a specialised training course, which will be the only additional resource employed to manage the patient profile, other than the time required for the medical consultation.

- The effectiveness of the intervention will be evaluated objectively, in terms of improved functional capacity and reduced incidence of frailty-related adverse events.

- One limitation of this study is that the measure of effectiveness obtained will be determined by the participants' adherence to the indications received and will require the health professionals involved to record the clinical history and to assess the participants' adherence to the intervention.

- The participants will not be representative of the entire population with frailty, as only persons sufficiently motivated to participate will be selected (although from a pragmatic standpoint this is an advantage in terms of external validity).

\section{INTRODUCTION}

Frailty is apparent as a progressive decline in age-related physiological systems, decreasing the reserve of intrinsic functional capacity and provoking extreme vulnerability to stressors, thus increasing the risk of adverse events. ${ }^{1}$

Persons with frailty are three to four times more likely to become dependent, with respect to robust persons of the same sex and comparable age. ${ }^{2}$ The prevalence of frailty varies according to the measurement 
instrument used and the scope of study. In this respect, among a population of persons aged over 65 years, $18 \%$ (95\% CI $15 \%$ to $21 \%$ ) presented frailty according to a recent (2017) meta-analysis of publications in 22 European countries. ${ }^{3}$

Frailty is an independent risk factor for the occurrence of adverse health events. Although it is not completely reversible, its degree of severity can be attenuated. In consequence, the approach taken to persons with this condition is of vital importance in the prevention of dependence. Research evidence has highlighted the effectiveness of interventions based on muscle strengthening through exercise, dietary improvements and the control of polypharmacy, among other aspects. ${ }^{4}$

However, the question of frailty is not systematically addressed in the primary healthcare (PHC) network, possibly due to a lack of consensus on the most appropriate instruments for identifying its presence among patients in PHC, or to the fact that reports on the effectiveness of interventions in this area often originate in areas of attention other than PHC, or because the necessary resources are not available. ${ }^{56}$

Despite these limitations, it is universally accepted that frailty should be addressed in PHC; see, for example, international reference documents published by the European Innovation Partnership on Active and Healthy Ageing $^{7}$ and the Integrated care for older people proposal by the WHO. ${ }^{8}$

\section{Rationale}

The necessary priority of frailty within the framework of health services research is acknowledged in the European Commission Report on Aging (2012), according to which reducing disability and dependence by means of appropriate measures aimed at combating frailty should be at the forefront of all health-oriented policies. ${ }^{9}$

Tackling frailty in PHC requires appropriate, viable interventions based on existing evidence. In recent years several clinical trials have been conducted regarding the management of frailty, considering various types of intervention, including physical activity intervention programming (with or without nutritional/protein supplementation), psychological interventions, the optimisation of medication, pharmacotherapy and multifaceted interventions, ${ }^{10}$ involving a combination of physical and/or nutritional activity together with psychosocial interventions, medication management or pharmacotherapy.

A single-centre clinical trial conducted in Sydney (Australia) evaluated the efficacy of an individualised interdisciplinary multifactorial intervention, with respect to the baseline frailty characteristics observed, versus placebo treatment. ${ }^{11}$ The intervention group was attended by a geriatrician, a rehabilitation physician, a nurse and a dietitian, coordinated by a physiotherapist. In the medium term (12 months), the patients in the intervention group achieved a reduction in the prevalence of frailty, and improvements in some of its components, such as walking speed and level of physical activity. In another single-centre clinical trial, conducted in Singapore among two populations, one presenting frailty and the other in a prefrail condition, the study population was randomised into four intervention groups (physical exercise, nutrition, cognitive training and a combination of these three), and a control group. Improvements (ie, reduced levels of frailty) were observed among all four intervention groups, in the short and medium terms (3, 6 and 12 months). The persons in the combined intervention group obtained the best results at 1 year, both in reduced frailty and in improved indicators of strength, speed and energy. ${ }^{12}$

Numerous studies have correlated frailty with the altered expression of genes related to oxidative stress, metabolism and inflammation. ${ }^{13-15}$ However, to our knowledge, no follow-up studies have been undertaken of these alterations following a physical or nutritional intervention, and therefore we consider it of interest to evaluate the effect produced on blood markers by a multifactorial intervention.

A recent systematic review of the effectiveness of interventions aimed at preventing the progression of frailty, while observing certain positive aspects, highlighted the low to moderate quality of the studies considered, especially in the sense that few studies have evaluated frailty both before and after the intervention. Moreover, in many cases the criteria employed were insufficiently robust. ${ }^{16}$ This systematic review also indicated the need for more economic evaluations of frailty interventions, in different decision-making contexts.

The main tools used in identifying persons with frailty are derived from the two theoretical frameworks that have been employed to develop the frailty construct. On the one hand, the phenotypic model of frailty ${ }^{14}$ in which a phenotype is obtained to represent the situation in which the risk of disability is apparent. This phenotype is determined by evaluating five signs and symptoms of the patient's physical sphere (exhaustion, low physical activity, slowness, weakness and shrinking).${ }^{14}$ The second model is that of Rockwood and Mitnitsky, whose frailty construct is based on the accumulation of deficits. The frailty index proposed by these authors contains 70 items, including disease, health status, geriatric syndrome and degree of disability. ${ }^{17}$ The two instruments differ in the dimensions evaluated, but in each case their calculation requires significant time and expertise. ${ }^{18}$ In addition, alternative means of assessing frailty status have been proposed, such as tests of physical performance or execution. These provide greater reproducibility and are less time consuming in the field of primary care. The most commonly used instruments of this type are the Timed Up and Go (TUG) test and the Short Physical Performance Battery (SPPB). ${ }^{19}$

The proposal described in this paper is based on an objective, reproducible and recognised measure for the identification of persons with frailty. Moreover, it is sensitive to change, thus reflecting the patient's long-term 
evolution, as recommended by a Spanish strategic document and by recent British guidelines in this area. ${ }^{20} 21$

We propose an intervention strategy that is expected to produce results with great potential for direct applicability in PHC. The research outcomes achieved will, in the near future, provide the scientific and healthcare community with a proposal for a viable, appropriate intervention in the PHC network of the national health system, without the need to provide additional resources, other than the time required for the medical consultation and the necessary complementary training for the health personnel involved in managing this type of patient profile.

\section{Objectives}

The main aim of this study is to evaluate the effectiveness of a multifactorial intervention (appropriateness of pharmaceutical prescription, nutritional care and exercise intervention) for persons with frailty, in terms of improving their functional capacity and reducing the incidence of frailty-related adverse events (dependence, falls, institutionalisation and hospitalisation) at 12 months' follow-up.

Secondarily, the effectiveness of the intervention will be evaluated in the following terms:

1. (A) Incidence of transition from frailty to robustness, at 6 and 12 months; (B) incidence of dependence and falls at 6 and 12 months; (C) incidence of institutionalisation at 6 and 12 months; (D) new hospitalisations at 6 and 12 months; (E) level of physical activity at 6 and 12 months; (F) prevalence of polypharmacy and potentially inappropriate prescriptions at 6 and 12 months; (G) prevalence of nutritional changes at 6 and 12 months; $(\mathrm{H})$ health-related quality of life at 6 and 12 months.

2. After the intervention, the expression of the molecular pattern associated with frailty will be validated and the complete transcriptome of each participant will be determined.

3. The efficiency (cost-effectiveness) of the intervention will be evaluated.

4. The viability and appropriateness of the intervention will be evaluated, via the qualitative exploration of barriers and facilitators.

\section{METHODS AND ANALYSIS \\ Design}

Pragmatic multicentre clinical trial, single blind, cluster randomised, with two arms: multifactorial intervention in PHC versus usual follow-up. The randomisation unit is the patient list and the analysis unit is the patient. In addition, a cost-effectiveness study and a qualitative study will be carried out, based on semistructured interviews and focus groups. The PRagmatic Explanatory Continuum Indicator Summary version 2 (PRECIS-2) instrument will be used before and after the fieldwork, to determine the degree of pragmatism of the trial, with blind assessment, followed by second-round agreement,

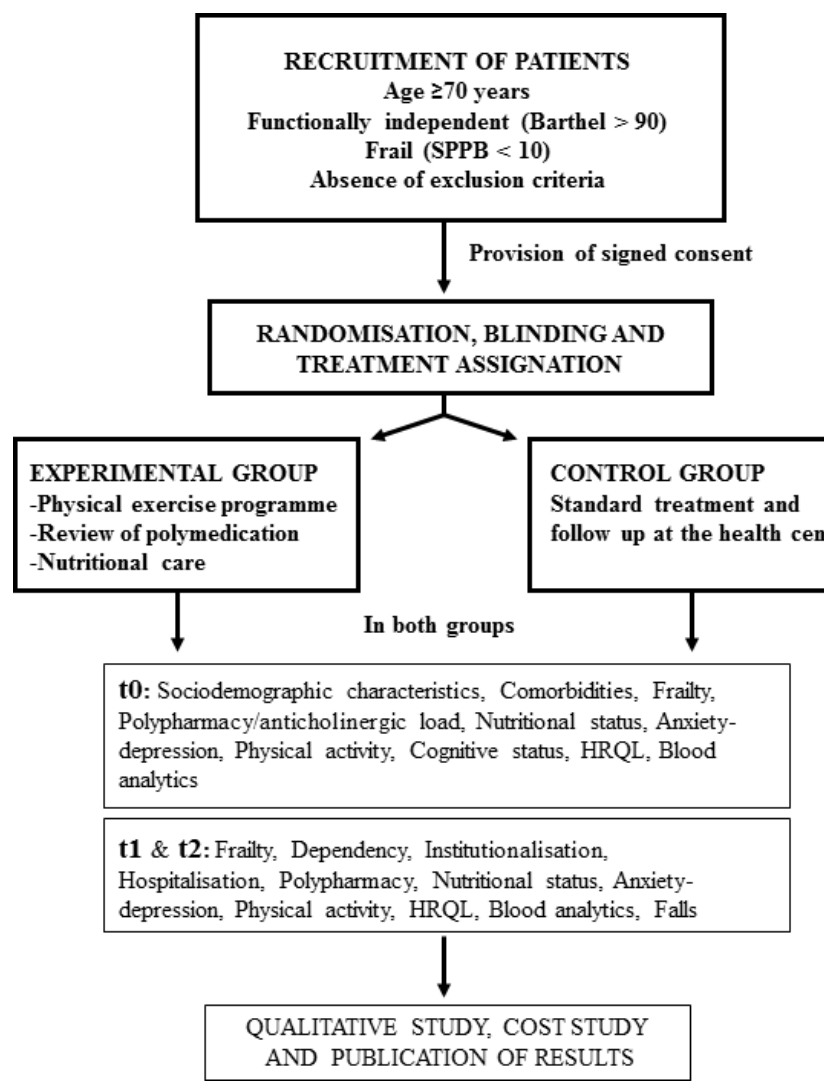

Figure 1 Study flow chart. HRQL, health-related quality of life; SPPB, Short Physical Performance Battery.

by three evaluators. ${ }^{22}$ The study design is illustrated in figure 1 .

\section{Setting}

The study will have a duration of 3 years (with a scheduled follow-up of 12 months). The recruitment start date will be in January 2020 and is expected to be completed 3 months later (March 2020). It will be conducted in two PHC areas in Spain, one in the south, at health centres in the Costa del Sol Primary Care District (reference population 137490 inhabitants), and the other in the north, in health centres of the Integrated Services Organisations of Gipuzkoa (reference population 175000 inhabitants), within the Basque Country Health System.

\section{Eligibility criteria}

The participants will be $\geq 70$ years of age, resident in the community and should meet the following criteria for frailty and functionally independent, respectively: SPPB score $<10$ points $^{20}$ and Barthel score $>90$ points. ${ }^{23}$ The SPPB will be used because this instrument identifies persons at risk of presenting adverse health outcomes (dependence, falls, institutionalisation and death). ${ }^{24-26}$ Furthermore, it is useful for the identification of frailty in clinical practice and has been recommended by the Spanish Health Ministry for this purpose (recommendation in which prefrail subjects are not taken into account).

Prior informed consent to participate will be obtained in all cases. Patients in a terminal condition (as defined 
in the guidelines published by the Spanish Palliative Care Society), persons not habitually residing in the study area (ie, for at least 6 months every year), persons with difficulties communicating in Spanish (or Basque, for those residents in the Basque Country) and persons diagnosed with cognitive impairment will be excluded from the study. This condition will be determined according to the MiniMental State Examination (MMSE), applying a threshold of MMSE <23 for cognitive impairment. ${ }^{27}$

\section{Sample size considerations and randomisation}

Kim $e t a l$, studying a population of frail elderly women, reported that an intervention based on physical exercise and diet achieved a reduction of 1.6 points (SD: 3 ) in the TUG test score, which was considered clinically significant. $^{28}$ In another study, the intraclass correlation coefficients (ICC) in the results obtained for PHC were less than $0.05,{ }^{29}$ and so for the purposes of the present study an ICC of 0.05 is assumed. A cluster size of five participants per patient list is believed realistic for a study of these characteristics. Therefore, at least 15 clusters (75 participants in total) should be included in each branch of the study. Assuming follow-up losses of about 25\%, 100 participants per branch (20 clusters of 5) will need to be recruited in order to detect the above-mentioned difference with a statistical power of $80 \%$ and a significance of $5 \%$. In each PHC area, 50 participants from the intervention branch and another 50 from the control branch will be recruited (see figure 1 , study process flow chart).

The randomisation unit will be the patient list and the analysis unit will be formed of persons aged $\geq 70$ years and presenting frailty. Randomisation by patient list cluster is considered appropriate as we wish to consider patient outcomes following treatment by the same physician.

\section{Recruitment}

We will consult with the physicians who took part in a previous study, which evaluated the predictive capacity of several instruments used to assess frailty-related adverse events. ${ }^{30}$ If the required number of doctors is not obtained, further patient lists will be sought. The lists corresponding to 40 family doctors who have expressed interest in participating in the study will be obtained and from each one, five patients will be recruited. Thus, for each list, all patients who meet the criteria of age $(\geq 70$ years) and level of autonomy (Barthel index $>90$ points) will be systematically screened, consecutively, when they attend the primary care centre for any reason. These patients will be seen by the research nurses taking part in the study and each patient's compliance or otherwise with the frailty criterion (SPPB $<10$ points) will be assessed. When frailty is established, the patient's informed consent will be requested, and if granted, the baseline assessment will be made. If consent is refused, the patient will be excluded from the study. The research nurse's evaluation of the inclusion criteria and the healthcare team's communication to the patients of the study group to which they are assigned will take place on different days, in order to avoid selection bias.

The randomisation procedure will be performed as follows: the patient lists will be allocated to the control or intervention group, randomly, once the medical professionals responsible for each list have agreed to participate in the project. The allocation will be carried out as randomisation by 'blocks'. By means of the 'sample' function of the $\mathrm{R}$ program of statistical analysis, in each region, half of the lists will be assigned randomly to the intervention group and the other half to the control group, simultaneously.

\section{Intervention}

The pragmatic clinical trial will have two study arms: an experimental group receiving a multicomponent intervention and a control group receiving usual care and follow-up at a PHC centre.

The scheduled inclusion period is 6 months with follow-ups at 6 and 12 months after the baseline assessment. The baseline assessment and the two follow-up assessments will be made both for the intervention group and for the control group. The nurses responsible for this operation will be trained accordingly and will be blind to the group assignment. In order to maintain the blinding, the health teams responsible will ask the patients not to tell the researchers the group to which they have been assigned. The assessments made will include questionnaires, anthropometric measurements and blood sampling. During the follow-up period, each patient's electronic clinical history will be obtained, to extract information on prescriptions (at baseline and any changes during follow-up), institutionalisation and hospitalisations.

The multicomponent intervention will be composed of the following elements:

A. Physical exercise: Based on WHO recommendations for physical activity by the elderly. ${ }^{31}$ The patients will be evaluated by their physicians, who will provide and describe the use of a table of recommended exercises. This consists of 12 muscle-strengthening exercises that are appropriate for the subject and can be performed without supervision. The patients will be advised of the number of repetitions that should be made and the frequency of performance. In addition, a followup plan will be provided. The patients will be advised to perform the exercise programme five times per week, and also to take three $30 \mathrm{~min}$ walks at moderate intensity every week. As no supervision is needed, the physical exercise programme is to be performed throughout the year of patient follow-up.

B. Review of polypharmacy: The patients' PHC doctor will review the medication prescribed in each case and give instructions for appropriate changes, if needed, according to the Screening Tool of Older Person's Prescriptions (STOPP) criteria. The Spanish version of the 2014 criteria will be used to detect potentially inappropriate prescriptions recorded on the baseline 
assessment of the patients in the experimental arm. ${ }^{32}$ Using the electronic medical record, data on all the drugs prescribed to each patient in the intervention group will be compiled, together with the dose, on the referral date. Drugs will be registered according to the name of the active ingredient. The clinical history of each patient assigned to the experimental group will be consulted to ensure compliance with the treatment criteria, according to the patient's clinical condition and the dose prescribed. The primary care physicians will then take the appropriate treatment decision, which will be recorded for subsequent evaluation. The medication prescribed to each participant will be reviewed at the beginning, after 6 months and at the end of the study.

C. Nutritional care: This component focuses on evaluating the quality of the patients' habitual diet and on detecting any risk of malnutrition (or its presence, and in this case, prescribing appropriate treatment), taking into account expert recommendations and following established nutritional guidelines for this population group. At baseline, the presence or absence of the risk of malnutrition and the quality of the habitual diet will be evaluated. The scores obtained by application of the Mini Nutritional Assessment-Short Form questionnaire will be used to classify patients as 'not at nutritional risk' (and given recommendations on healthy eating), 'at risk of malnutrition' (and given specific advice on nutrients that should be included in the daily intake of food) or 'malnourished' (and given a personalised nutrition plan, which may include texture modification). ${ }^{33}$ The patients' nutritional status and the introduction of corrective measures if malnutrition (or risk of malnutrition) is detected will be determined in the baseline evaluation.

Using the information compiled at baseline, the doctor and the nurse responsible for each participant in the intervention group will jointly determine the level of intervention necessary, and will record in the patient's clinical history any changes effected in the pharmaceutical prescription and the recommendations made regarding exercise and diet, both at the outset and in subsequent follow-up visits. The corresponding doctor-and-nurse team will assess the patient's adherence to treatment at the 6 and 12 months' follow-up visits.

The doctors and nurses collaborating in the fieldwork for this project will take a specialised training course, accredited by the corresponding quality agency in each autonomous community, after they have been randomised and before the recruitment of participants begins. This training will only be received by the doctors and nurses assigned to the intervention group. The course will consist of four sessions. The first will be a faceto-face session to present the study and the tools that will be used to detect frailty among the elderly, based on the Barthel index of autonomy and the SPPB tests of frailty. The remaining three sessions will be offered in e-learning format and will focus, in turn, on each of the elements of the intervention (physical exercise, review of polypharmacy and nutritional intervention). The training modules will be imparted by a member of the research team who has expert knowledge of the corresponding area (ie, by a physiotherapist, a pharmacist and a nutritionist, respectively). The e-learning format ensures consistency of instruction in each of the autonomous communities in which the intervention will take place.

Usual care will be provided on demand in the control group, due to the chronic pathology presented, since the Spanish national health system has no specific plans for managing patients with frailty.

All participants will be followed up in primary care, at the baseline evaluation, and after 6 and 12 months. In the experimental group, we expect the patients to obtain an improvement in functional capacity and in preventing adverse events that may impair the quality of life. The study involves no risks beyond the discomfort derived from the extraction of blood at the three time points established for evaluation.

\section{Outcome measures}

\section{Primary outcome measure}

The effectiveness of the multicomponent intervention in participants presenting frailty, in terms of improved functional capacity and reduced incidence of adverse frailtyrelated events (dependency, falls, institutionalisation and hospitalisation), will be ascertained after 12 months' follow-up.

Functional capacity will be measured by the TUG test. Participants with low functional capacity will be identified by the failure to achieve the cut-off point of $12 \mathrm{~s}$, a criterion recommended in previous studies with communitydwelling older people. ${ }^{34} 35$

With respect to the incidence of adverse events, a score less than or equal to 90 points in the Barthel test will be considered to represent dependency. Other adverse events assessed during the follow-up year will be institutionalisation and hospitalisations (by number, hospital service required, type-programmed or urgent-and duration).

\section{Secondary outcome measures}

(1) Incidence of transitions from frailty to robustness at 6 and 12 months. Patients will be classed as robust if they obtain a score greater than or equal to 10 in the SPPB test; (2) Incidence of dependency and falls at 6 and 12 months; (3) Incidence of institutionalisation at 6 and 12 months; (4) New hospitalisations at 6 and 12 months; (5) Level of physical activity at 6 and 12 months. This parameter will be evaluated using a scale adapted from the WHO recommendations on physical activity for elderly people ${ }^{31}$; (6) Prevalence of polypharmacy and potentially inadequate prescriptions at 6 and 12 months. The number of STOPP criteria met will be determined for the three time points (baseline and at 6 and 12 months of follow-up); (7) Prevalence of nutritional alterations at 6 and 12 months. Alteration is defined as a nutritional score 
of 17-23.5 points, and malnutrition as a score lower than 17 points in the Mini Nutritional Assessment. Body mass index will be measured and a food frequency questionnaire will be used; (8) Health-related quality of life at 6 and 12 months, evaluated using EuroQoL 5 Dimensions 5 Levels (EQ-5D-5L) ${ }^{36}$; (9) Depression at 6 and 12 months; (10) After the intervention, the expression of the molecular pattern associated with frailty and the complete transcriptome will be determined for each participant; (11) The efficiency of the intervention (cost-effectiveness); (12) The feasibility and appropriateness of the intervention, via an exploration of barriers and facilitators, using a qualitative approach.

\section{Adjustment variables}

Age; sex; education background; income; cohabitation status; social network, on the Lubben $\mathrm{Scale}^{37}$; comorbidity: active diagnoses and Charlson Comorbidity Index ${ }^{38}$; cognitive status, according to the $\mathrm{MMSE}^{27}$; and alterations in affective relations, according to the Goldberg Scale. ${ }^{39}$

The costs incurred in providing the two study arms, including intervention costs, will be evaluated by the health services concerned. The resources employed for each cost component will be identified from the patients' electronic medical records. Unit costs will be identified from standard sources, according to availability. This calculation will be based on the number of follow-up consultations required for each participant in primary and specialised care. No additional cost is assumed for the intervention, as it will be performed by the usual personnel, although after specific training.

The expression of the molecular pattern associated with frailty after the intervention will be validated, and the complete transcriptome of the participants will be determined using Affymetrix microarrays.

\section{Data collection and management}

The baseline assessment and the follow-up assessments at 6 and 12 months will be performed, both for the intervention group and the control group, at a prearranged appointment with the nursing staff (trained for this task and blind to the group assignment), at the health centre. These assessments will be based on questionnaires, anthropometric measurements and blood samples. Throughout the follow-up period, information on prescriptions (at baseline and any changes that may occur), institutionalisation and hospitalisations will be obtained from the electronic medical record.

\section{Statistical analysis}

For the descriptive analysis of the data, the categorical variables will be represented by frequencies and percentages; the continuous variables, by the mean and SD when the distribution is normal, and by the median and the first and third quartiles otherwise. The differences between these variables will be measured by the $\chi^{2}$ test for independent categorical variables and by the McNemar's test for dependent variables. For continuous variables with a normal distribution, Student's t-test will be applied for paired data and related samples; for continuous variables that do not follow a normal distribution, Wilcoxon's non-parametric test, the sum of ranges for independent variables and ranges with signs for related variables will be used. In addition, longitudinal models per intention to treat will be generated to observe the effect of the intervention over time. Given the hierarchical structure of the data, mixed regression models will be considered, adjusted for confounding variables, and the interaction between the treatment and the time elapsed will be studied, considering the clusters and individuals as random effects. For the dichotomous variables, logistic regressions will be considered and for the continuous ones, linear regressions. In all analyses, the level of significance used will be $\mathrm{p}<0.05$ and the main outcome variables with their respective $95 \%$ CIs will be described. All analyses will be performed using the SAS program version 9.3 or the free software R.

The cost-effectiveness measure used will be the incremental cost per quality-adjusted life-year (QALY). The QALYs will be calculated from the health-related quality of life information collected during the study, using the EQ-5D-5L questionnaire. This information will be obtained at the beginning of the study, and at 6 and 12 months' follow-up for each patient. Patient-specific utility profiles will be constructed, assuming a linear relationship between the EQ-5D-5L scores obtained at each follow-up point. The cost-effectiveness ratio will be calculated as the incremental cost-effectiveness ratio (ICER), dividing the differences in costs by the differences in the observed effects. Non-parametric methods will be used to calculate the ICER CIs based on bootstrap estimates of average costs and the difference effects, which will also be used to construct a cost-effectiveness acceptability curve. As is common for this type of intervention in the Spain, we assume a willingness to pay of $€ 25.000$ per QALY. ${ }^{40}$

\section{Qualitative study}

Once the intervention has concluded, a focus group meeting will be held with the participating medical staff (three physicians and three nurses) for each PHC area. These meetings will be audio/video recorded for subsequent transcription. There will also be at least six semistructured interviews with medical personnel and the patients in the intervention group. Each interview will be recorded and then transcribed in order to perform a content analysis. Boxes 1 and 2 detail the scripts to be employed with medical personnel and patients, respectively.

The qualitative data analysis will be conducted using the Taylor-Bogdan system, focusing on data preparation, discovery of emerging issues, coding, interpretation, relativisation and determination of methodological rigour. For these purposes, NVivo V.11 software will be used. The categories and subcategories will be determined according to the topics that the researcher considers appropriate. Two types of category, aprioristic and emergent, will be 
Box 1 Script for semistructured interviews with medical personnel

1. Has it been easy, within your professional area of responsibility, to carry out this project? Has it meant more work or greater personal effort?

2. At a professional level, what have you derived from participating in this study? And on a personal level?

3. In your opinion, what are the methodological weaknesses and strengths of this study? What would you change if you could start again from scratch?

4. What barriers have you encountered during your work in this study? Who have been the facilitating agents that have helped you? Tell me a little about these questions.

5. What degree of importance do you grant to this project? Do you think that the interventions made should be incorporated into standard protocols? Why?

6. In your view, what factors will determine whether or not these measures are implemented in the future? Do you think that other healthcare professionals would agree? Why?

7. Do you think this project is beneficial to health service users and to the medical personnel? Why?

8. Have you noticed any differences in the users following their participation in the interventions? Has there been a 'before and after'? In what respects? Why do you think this is?

9. Do you think these patients will make less use of health service resources after the intervention? Tell me a little about this question.

10. Would you like to add anything else?

\section{Box 2 Script for semistructured interviews with patients}

1. When you attended the health centre, how did you find the waiting time? How did you feel about this?

2. Was there any factor during the wait that you found annoying (such as noise, lighting, the number of people around)?

3. What would you improve during the waiting time to feel more relaxed and comfortable?

4. How do you consider the experience of participating in this project? Would you participate again?

5. Does your health feel better than before you started in this project? Why?

6. Do you think you needed such specific attention in order to feel better? In what way did this attention help?

7. Have you noticed any change in your daily routine? Do you feel better about yourself? Tell me a little about the changes you have experienced.

8. Do you think that the treatment received from the medical people involved was appropriate? How did you feel about these persons?

9. Can you suggest any ways in which the interventions might be improved?

10. Have your nutritional habits changed in any way? Has the way you eat or the foods you eat changed? Tell me a little about this.

11. Do you take more physical exercise now than before the project? Did you do all the exercises that were recommended? Did you find them easy? Would you change anything in this respect?

12. Regarding the medication you were taking before the intervention, have you reduced the number of drugs you take? Do you feel better? Do you think you are doing the right thing?

13. In general, how would you rate this initiative?

14. Would you like to add anything else? distinguished. The a priori categories will be constructed before the information gathering process, and the emergent ones as they arise during the research process. In this case, the reference available will be that of the emergent categorisation system. ${ }^{42}$

For each study group, a minimum of eight participants will be recruited by purposive selection, stratifying by sex, PHC area and degree of adherence to the study. New subjects will be selected until information saturation is achieved.

In qualitative research, the analysis of prior information can lead to data collection techniques being reconsidered if the study goals are not achieved. Therefore, the data collection process and its subsequent analysis will be considered to have concluded when the information is complete and the study goals attained.

\section{ETHICS}

This research project has been approved by the Euskadi Scientific Research Ethics Committee (CEIm-E_Versión DEF 25.03.2019_Acta 07/2019, 22 May 2019) and by the Costa del Sol Research Ethics Committee (007_mar19_ PI-Intervención Fragilidad, 28 March 2019).

The present study will at all times adhere to the Declaration of Helsinki and be conducted in accordance with Spanish legislation regulating patient autonomy and rights and obligations regarding clinical information and documentation. No clinical data will be collected other than those listed above. Moreover, all data collected in this project will be recorded anonymously, in strict accordance with the laws and data protection regulations in force (Organic Law 3/5 December 2018 on the Protection of Personal Data and Guarantee of Digital Rights). All patients recruited to this study will sign an informed consent form prior to their participation in the clinical trial. In addition, the healthcare personnel and the patients in the intervention group will sign a specific consent form to participate in the qualitative phase (semistructured interview).

No payment will be made to the healthcare professionals or to the patients involved in any phase of the study.

\section{Dissemination}

Following completion of the study, the principal investigator is expected to publish the results of this research in a peer-reviewed open access scientific journal. Trial investigators have the right and responsibility to communicate their findings to the scientific community and to the public. Findings of the trial will also be presented at national and international meetings of relevant professional bodies and research groups.

These findings are expected to be available when the study is finished and the data are ready to be analysed. Requests to access data will be subject to participant confidentiality concerns and to current European Commission guidance on data-sharing plans. 


\section{Implications and contribution}

The fundamental aim of this proposal is to contribute further evidence on the approach adopted to persons with frailty. In this respect, we propose a multicentre study, to be conducted in the context of PHC. This study will require no resources other than those usually present in this field. It will measure the effectiveness of a multifactorial intervention in terms of standard care practice parameters (appropriateness of prescription, nutritional care, exercisebased intervention, and so on) for persons with frailty. The methodology described is robust (controlled clinical trial) and includes a multicriteria evaluation process. The study is expected to contribute to enhancing the intrinsic functional capacity of persons with frailty.

\section{Author affiliations}

${ }^{1}$ Unidad de Investigación, Agencia Sanitaria Costa del Sol, Marbella, Málaga, Spain ${ }^{2}$ Red de Investigación en Servicios de Salud en Enfermedades Crónicas (REDISSEC), Madrid, Spain

${ }^{3}$ Instituto de Investigación Sanitaria Biodonostia, Grupo de Atención Primaria, San Sebastián, Spain

${ }^{4}$ Instituto de Investigación en Servicios de Salud Kronikgune, Baracaldo, Spain ${ }^{5}$ Unidad Gestión Clínica de Prevención, Promoción y Vigilancia de la Salud, Distrito Sanitario de Atención Primaria Costa del Sol, Mijas, Málaga, Spain

${ }^{6}$ Osakidetza, Centro de salud de Altza, San Sebastián, Spain

${ }^{7}$ Área del Medicamento, Distrito Sanitario de Atencion Primaria Costa del Sol, Mijas, Spain

${ }^{8}$ Servicio de Medicina Interna, Agencia Sanitaria Costa del Sol, Marbella, Spain

Collaborators InFrAP Investigators-Group 1. Instituto de Investigación Sanitaria Biodonostia: Itziar Vergara Mitxeltorena, Mónica Machón Sobrado, Maider Mateo Abad, Kalliopi Vrotsou, Miren Revuelta Aramberri. Centro de Salud de Altza: Carolina Güell Pelayo. Centro de Salud de Beraun: Ana Isabel Diez Ruiz. Centro de Salud Amara Berri: Irati Rodriguez Matesanz. Fundación Matía: Iván Antón Rodrigo; Group 2. Hospital Costa del Sol: FRR, RQL, Ana Isabel Calderón Durán, Jimena Abiles 0sinaga, María Padilla Ruiz. Distrito Sanitario Costa del Sol: ECF, LBR, María Dolores Llamas del Castillo, Felipe Salas Samper, María Antonia Nava del Val, Susana Clavero Cano, Sonia María Lozano Gómez, Isabel María Rodríguez Jiménez. Fundación Canaria de Investigación Sanitaria (FUNCANIS): Beatriz León Salas.

Contributors IV, FRR and MM were responsible for the conceptualisation of the research question, approach and rationale. IV and FRR were responsible for sought funding and ethical approval. MM, MMA, KV and LBR developed the methods to be used for this protocol. IV, FRR, MM, ECF, CG and RQL provided initial research into existing literature and developed the introduction to this manuscript. FRR prepared the first draft of this manuscript, which was reviewed and revised by MM and IV. All authors read and approved the final manuscript.

Funding This study was supported by grants from the Instituto de Salud Carlos III jointly funded by the European Fund for Regional Development-FEDER, with grant numbers PI18/01558 and PI18/01177.

Competing interests None declared.

Patient consent for publication Not required.

Provenance and peer review Not commissioned; externally peer reviewed.

Open access This is an open access article distributed in accordance with the Creative Commons Attribution Non Commercial (CC BY-NC 4.0) license, which permits others to distribute, remix, adapt, build upon this work noncommercially, and license their derivative works on different terms, provided the original work is properly cited, appropriate credit is given, any changes made indicated, and the use is non-commercial. See: http://creativecommons.org/ licenses/by-nc/4.0/.

\section{ORCID iDs}

Francisco Rivas-Ruiz http://orcid.org/0000-0002-8894-0501

Itziar Vergara http://orcid.org/0000-0001-9671-7898
REFERENCES

1 Cesari M, Prince M, Thiyagarajan JA, et al. Frailty: an emerging public health priority. J Am Med Dir Assoc 2016;17:188-92.

2 Kojima G. Frailty as a predictor of future falls among communitydwelling older people: a systematic review and meta-analysis. J Am Med Dir Assoc 2015;16:1027-33.

3 O'Caoimh R, Galluzzo L, Rodríguez-Laso Ángel, et al. Prevalence of frailty at population level in European advantage joint action member states: a systematic review and meta-analysis. Ann Ist Super Sanita 2018;54:226-38.

4 Morley JE, Vellas B, van Kan GA, et al. Frailty consensus: a call to action. J Am Med Dir Assoc 2013;14:392-7.

5 Walston J, Buta B, Xue Q-L, et al. Frailty screening and interventions: considerations for clinical practice. Clin Geriatr Med 2018;34:25-38.

6 Pialoux T, Goyard J, Lesourd B. Screening tools for frailty in primary health care: a systematic review. Geriatr Gerontol Int 2012;12:189-97.

7 Cano A, Dargent G, Carriazo A, et al. Tackling frailty and functional decline: background of the action group A3 of the European innovation partnership for active and healthy ageing. Maturitas 2018;115:69-73.

8 de Carvalho IA, Epping-Jordan J, Beard JR. Integrated care for older people, 2019.

9 European Commission. The 2012 ageing report: economic and budgetary projections for the EU27 member states (2010- 2060. Brussels, 2012.

10 Negm AM, Kennedy CC, Thabane L, et al. Management of frailty: a protocol of a network meta-analysis of randomized controlled trials. Syst Rev 2017;6:130.

11 Cameron ID, Fairhall N, Langron C, et al. A multifactorial interdisciplinary intervention reduces frailty in older people: randomized trial. BMC Med 2013;11:65.

$12 \mathrm{Ng}$ TP, Feng L, Nyunt MSZ, et al. Nutritional, physical, cognitive, and combination interventions and frailty reversal among older adults: a randomized controlled trial. Am J Med 2015;128:1225-36.

13 Rodríguez Mañas L. Determinants of frailty and longevity: are they the same ones? Nestle Nutr Inst Workshop Ser 2015;83:29-39.

14 Fried LP, Tangen CM, Walston J, et al. Frailty in older adults: evidence for a phenotype. J Gerontol A Biol Sci Med Sci 2001;56:M146-57.

15 Walston J, McBurnie MA, Newman A, et al. Frailty and activation of the inflammation and coagulation systems with and without clinical comorbidities: results from the cardiovascular health study. Arch Intern Med 2002;162:2333-41.

16 Apóstolo J, Cooke R, Bobrowicz-Campos E, et al. Effectiveness of interventions to prevent pre-frailty and frailty progression in older adults: a systematic review. JBI Database System Rev Implement Rep 2018;16:140-232.

17 Rockwood K, Song X, MacKnight C, et al. A global clinical measure of fitness and frailty in elderly people. CMAJ 2005;173:489-95.

18 Belloni G, Cesari M, Frailty CM. Frailty and intrinsic capacity: two distinct but related constructs. Front Med 2019;6:133.

19 Cesari M, Landi F, Calvani R, et al. Rationale for a preliminary operational definition of physical frailty and sarcopenia in the SPRINTT trial. Aging Clin Exp Res 2017;29:81-8.

20 Inter-territorial Council of the National health system. Consensus document onfrailty and falls prevention among the elderly. The Prevention and Health Promotion Strategy of the Spanish NHS, 2014.

21 Turner G, Clegg A, et al, British Geriatrics Society. Best practice guidelines for the management of frailty: a British geriatrics Society, age UK and Royal College of general practitioners report. Age Ageing 2014;43:744-7.

22 Loudon K, Treweek S, Sullivan F, et al. The PRECIS-2 tool: designing trials that are fit for purpose. BMJ 2015;350:h2147.

23 González N, Bilbao A, Forjaz MJ, et al. Psychometric characteristics of the Spanish version of the Barthel index. Aging Clin Exp Res 2018;30:489-97.

24 Pritchard JM, Kennedy CC, Karampatos S, et al. Measuring frailty in clinical practice: a comparison of physical frailty assessment methods in a geriatric out-patient clinic. BMC Geriatr 2017;17:264.

25 Lauretani F, Ticinesi A, Gionti L, et al. Short-Physical performance battery (SPPB) score is associated with falls in older outpatients. Aging Clin Exp Res 2019;31:1435-42.

26 Guralnik JM, Simonsick EM, Ferrucci L, et al. A short physical performance battery assessing lower extremity function: association with self-reported disability and prediction of mortality and nursing home admission. J Gerontol 1994;49:M85-94.

27 Lobo A, Saz P, Marcos G, et al. [Revalidation and standardization of the cognition mini-exam (first Spanish version of the Mini-Mental 
Status Examination) in the general geriatric population]. Med Clin 1999:112:767-74

$28 \mathrm{Kim} \mathrm{H}$, Suzuki T, Kim M, et al. Effects of exercise and milk fat globule membrane (MFGM) supplementation on body composition, physical function, and hematological parameters in community-dwelling frail Japanese women: a randomized double blind, placebo-controlled, follow-up trial. PLoS One 2015;10:e0116256.

29 Campbell M, Grimshaw J, Steen N, et al. Sample size calculations for cluster randomised trials. J Health Serv Res Policy 2000;5:12-16.

30 Vergara I, Rivas-Ruiz F, Vrotsou K, et al. Validation and comparison of instruments to identify frail patientes in primary care settings: study protocol. BMC Health Serv Res 2016;16:354

31 World Health Organization. Global recommendations on physical activity for health 65 years and above, 2011. Available: https://www. who.int/dietphysicalactivity/publications/recommendations65yea rsold/en/ [Accessed 20 Nov 2019].

32 Delgado Silveira E, Montero Errasquín B, Muñoz García M, et al. [Improving drug prescribing in the elderly: a new edition of STOPP/ START criteria]. Rev Esp Geriatr Gerontol 2015;50:89-96.

33 de Luis DA, López Mongil R, González Sagrado M, et al. Evaluation of the mini-nutritional assessment short-form (MNA-SF) among institutionalized older patients in Spain. Nutr Hosp 2011;26:1350-4

34 Herman T, Giladi N, Hausdorff JM. Properties of the 'timed up and go' test: more than meets the eye. Gerontology 2011;57:203-10.
35 Bischoff HAet al. Identifying a cut-off point for normal mobility: a comparison of the timed 'up and go' test in community-dwelling and institutionalised elderly women. Age Ageing 2003;32:315-20.

36 Hernandez G, Garin O, Pardo Y, et al. Validity of the EQ-5D-5L and reference norms for the Spanish population. Qual Life Res 2018;27:2337-48.

37 Lubben J, Blozik E, Gillmann G, et al. Performance of an abbreviated version of the Lubben social network scale among three European community-dwelling older adult populations. Gerontologist 2006:46:503-13.

38 Charlson ME, Pompei P, Ales KL, et al. A new method of classifying prognostic comorbidity in longitudinal studies: development and validation. J Chronic Dis 1987;40:373-83.

39 Montón C, Pérez Echeverría MJ, Campos R, et al. [Anxiety scales and Goldberg's depression: an efficient interview guide for the detection of psychologic distress]. Aten Primaria 1993;12:345-9.

40 Campillo-Artero C, Ortún V. Cost-Effectiveness analysis: why and how. Rev Española Cardiol 2016;69:370-3.

41 López Bastida J, Oliva J, Antoñanzas F, et al. [A proposed guideline for economic evaluation of health technologies]. Gac Sanit 2010;24:154-70.

42 Driessnack M, Sousa VD, Mendes IAC. An overview of research designs relevant to nursing: Part 2: qualitative research designs. Rev Lat Am Enfermagem 2007;15:684-8. 